\title{
Rejoinder on: Farkas' lemma: three decades of generalizations for mathematical optimization
}

\author{
N. Dinh • V. Jeyakumar
}

Published online: 1 February 2014

(C) Sociedad de Estadística e Investigación Operativa 2014

We would first like to express our gratitude to the colleagues for accepting the invitation of the Editor-in-Chief, TOP, Prof Miguel Goberna, to comment on the paper. We are extremely grateful to them for thoroughly reading the paper, preparing their insightful comments on it and adding breadth and depth to it.

We would also like to extend our warmest thanks to Prof Miguel Goberna for his kind invitation to write a survey on Farkas' lemma and to acknowledge the extremely successful research collaborations that we enjoyed with Prof Miguel Goberna over many years on the topic area of the paper.

At the outset, it is extremely pleasing to state here that all discussants have outstanding research expertise with major contributions to the field of mathematical optimization. Their different perspectives on mathematical optimization contribute to valuable discussions on some aspects of Farkas' lemma that may not have been fully covered in the original paper and thus provide an excellent complement. We feel now confident that the readers of the TOP will appreciate these discussions because the discussants have succeeded in further explaining the significance of Farkas' lemma to

This rejoinder refers to the comments available at doi:10.1007/s11750-014-0315-2; doi:10.1007/ s11750-014-0316-1; doi:10.1007/s11750-014-0317-0; doi:10.1007/s11750-014-0318-z.

Research was partially supported by grants from the Australian Research Council and from the NAFOSTED Vietnam.

N. Dinh $(\varangle)$

Department of Mathematics, International University, Vietnam National University-Ho Chi Minh City, Ho Chi Minh City, Vietnam e-mail: ndinh02@yahoo.fr

V. Jeyakumar

Department of Applied Mathematics, University of New South Wales, Sydney 2052, Australia

e-mail: v.jeyakumar@unsw.edu.au 
mathematical optimization in a wider perspective for the readers. In this rejoinder, we shall briefly elaborate on these discussions by category.

\section{Historical account of Farkas' lemma}

The brief comment by Boris Mordukhovich on the original Farkas' papers shows the historical significance of Farkas' lemma to the solution of a certain mechanical problem and to the derivation of Karush-Kuhn-Tucker optimality conditions. It highlights a fascinating account of the work of Gyula Farkas and also serves as a valuable addition to the readers.

\section{Sufficient conditions for generalized Farkas' lemmas}

The sufficient conditions for the generalization of Farkas' lemma form the main basis for the most of the discussants' comments. It is not a surprise because they determine the strength of the generalization whose implications extend to many areas of applications in mathematical optimization.

There are two types of sufficient conditions that are employed for generalizing Farkas' lemma: (1) primal conditions that are often expressed as a generalized Slater condition (2) dual conditions that appear in the form of a closed cone condition or an epigraphical condition. The link between these two types of conditions is important. Chong Li and Kung Fu Ng have clearly illustrated their links in their discussions for general cone-convex systems. It is of particular interest to note that the dual conditions are often weaker sufficient conditions than the primal conditions for a generalized Farkas' lemma. Moreover, the dual sufficient conditions of type (2), especially epigraphical conditions, are also necessary for certain generalizations of Farkas' lemma.

\section{Constraint qualifications in mathematical optimization}

The sufficient conditions for generalized Farkas' lemma lead to the so-called constraint qualifications in mathematical optimization. They are critical, in particular, for the validity of the associated powerful duality theory in mathematical optimization and for the development of optimality conditions for identifying optimal solutions of continuous optimization problems.

A class of closed cone sufficient conditions, called Farkas-Minkowski property and locally Farkas-Minkowski property, was developed by Miguel Goberna and Marco Lopez for studying generalizations of Farkas' lemma for semi-infinite inequality systems. Consequently, they used the corresponding constraint qualifications for treating semi-infinite optimization.

Marco Lopez has given a brilliant discussion on these conditions and their connections with other related constraint qualifications. In particular, he points out the interesting relationship between this class of constraint qualifications and the strong conical hull intersection (strong chip) property which was not considered in the paper. Indeed, this basic property, which is one of the central ingredients in the study of con- 
strained interpolation and best approximation [see Jeyakumar and Mohebi (2005)], has been shown to be the key characterizing property for optimality and strong duality of convex programming problems Jeyakumar (2006).

Nonlinear versions of the Farkas-Minkowski properties play a prominent role in nonconvex infinite and conic mathematical optimization problems as described by Boris Mordukhovich in his comments.

\section{Explicit applications of generalized Farkas' lemmas to mathematical optimization}

In our survey, a great deal of attention has been focussed on the direct and explicit applications of Farkas' lemma and its generalizations to problems of mathematical optimization. This is elegantly illustrated for linear semi-infinite optimization by Marco Lopez in his comments in terms of a two-step process for deriving optimality conditions. This two-step process continues to hold for many of the applications of Farkas' lemma and its generalizations for deriving optimality conditions for continuous optimization problems.

\section{Implicit roles of generalized Farkas' lemma in mathematical optimization}

Farkas' lemma and its generalizations also play important implicit roles in many areas of mathematics, including stability theory, variational analysis and generalized differentiation, set containment problems and large-scale data classification.

Boris Mordukhovich has provided a detailed account of implicit roles of generalized Farkas' lemma in the studies of Lipschitzian stability, second-order variational calculus. These roles are of particular importance in the calculation of various normal cones which are critical for the development useful optimality conditions for many classes of nonsmooth optimization problems Jeyakumar and Luc (2008). Xiaoqi Yang and Kaiwen Meng also briefly indicated similar implicit roles of Farkas' lemma and its generalizations in mathematical optimization.

Further implicit application of Farkas' lemma for classifying large-scale nonseparable data has been given by Mangasarian (2002) where Farkas' lemma was used for describing the containment of one set in another in terms of systems of linear inequalities. Since then several applications of Farkas' lemma and its generalizations have been given for obtaining more general set containment characterizations [see Goberna and Verda de Serio (2008) and other references therein].

\section{References}

Goberna MA, Verda de Serio VN (2008) On the stable containment of two sets. J Glob Optim 41:613-624 Jeyakumar V, Mohebi H (2005) Limiting epsilon-subgradient characterizations of constrained best approximation. J Approx Theory 135(2):145-159

Jeyakumar V (2006) The conical hull intersection property for convex programming. Math Progr Ser A 106:81-92 
Jeyakumar V, Luc DT (2008) Nonsmooth vector functions and continuous optimization. Springer, New York

Mangasarian OL (2002) Set containment characterization. J Glob Optim 24:473-480 